\title{
20 Transdisziplinäre Entwicklung eines Modells für das strategische Kommunikationsmanagement
}

\begin{abstract}
Ausgehend von Problemstellungen der Praxis ist am IAM Institut für Angewandte Medienwissenschaft ein Modell entwickelt worden, das die Kernprozesse des Kommunikationsmanagements im Spannungsfeld von Unternehmensführung, Organisation und Öffentlichkeit verortet.
\end{abstract}

\section{Herausforderungen des Kommunikationsmanagements aus Sicht der Praxis}

Das Verbinden von Kommunikations- und Unternehmensstrategie, das Schaffen von Vertrauen ins Unternehmen, ${ }^{1}$ die Positionierung und Vernetzung der Kommunikationsverantwortlichen innerhalb des Unternehmens und die Verarbeitung des ständig wachsenden Informationsflusses werden seit Jahren als die zentralen Herausforderungen des Kommunikationsmanagements gesehen. Diese Themen erscheinen denn auch im European Communication Monitor - der jährlich in ganz Europa durchgeführten Online-Befragung zur aktuellen Praxis und Entwicklung strategischer Kommunikation - seit 2008 konstant ganz oben auf der Liste der wichtigsten zukünftigen Issues der befragten Kommunikationsverantwortlichen (vgl. Zerfass et al., $2008 \mathrm{ff}$.) und verweisen deutlich auf die unterschiedlichen Kontexte, in denen das Kommunikationsmanagement agiert: Es steht in engem Bezug sowohl zur Unternehmensführung als auch zur Öffentlichkeit im Markt und im gesellschaftspolitischen Umfeld (Zerfass, 2010: 289), ist aber zugleich auch Teil der Organisationskommunikation, die alle Kommunikationsprozesse „in, von und um (über) Organisationen“ (Weder, 2010: 17) umfasst.

Während in der Praxis noch bis Ende der 1990er-Jahre klar getrennt wurde zwischen dem Marketing, das für die Ausrichtung des Unternehmens und seiner Kommunikation gegenüber dem Markt zuständig ist, und Public Relations, die

1 Mit Unternehmen werden im Folgenden nicht nur Wirtschaftsunternehmen, sondern Organisationen generell bezeichnet.

DOI 10.1515/9783110496604-021, (c) BY-NC-ND @ 2017 Nicole Rosenberger, Markus Niederhäuser, published by De Gruyter. This work is licensed under the Creative Commons Attribution-NonCommercialNoDerivs Beq bicgesellt von | ZHAW Zuercher Hochschule für Angewandte Wissenschaften 
sich auf das gesellschaftliche Umfeld ausrichten, so verschwimmt diese strikte Unterscheidung zwischen betriebswirtschaftlicher und kommunikationswissenschaftlicher Ausrichtung von Kommunikation seither zunehmend. Dies zeigt sich etwa in der Tendenz zur Reorganisation von Kommunikationsabteilungen in Richtung themenfokussierte Newsroom-Organisation, die die klassische Strukturierung nach Zielgruppen, Aufgaben und/oder Kanälen je länger, je mehr aufbricht. Im selben Zeitraum etablierte sich auf Stufe Unternehmensführung der Anspruch, dass sich sämtliche Kommunikationsfunktionen, also Marketingkommunikation und Public Relations, strategisch auf die Unternehmenspolitik auszurichten und einen Beitrag zur Wertschöpfung zu leisten haben, beispielsweise durch Reputationserhalt respektive -steigerung (Einwiller, 2014: 372).

Reputation ist jedoch kein fester Wert, sondern wird in einer ausdifferenzierten, medialisierten und skandalisierenden Gesellschaft, die von Wertepluralismus geprägt ist, ständig neu verhandelt (Eisenegger, 2005: 201). Die von den Kommunikationsverantwortlichen geäusserten Herausforderungen sind denn auch vor dem Hintergrund der unterschiedlichen und zum Teil divergierenden Erwartungen und Logiken von Unternehmensführung, Organisation und Öffentlichkeit zu sehen.

\section{Theoretische Perspektiven auf das Kommunikationsmanagement}

Die aus Sicht der Praxis beschriebenen Herausforderungen spiegeln sich auch in der Theoriebildung. Einerseits sind verschiedene organisationsbezogene Theorien und Modelle entwickelt worden, die das aufeinander Beziehen und Verschränken der verschiedenen Kommunikationsdisziplinen reflektieren (vgl. bspw. Zerfass, 2010; Bruhn, 2014). Andererseits hat der Einbezug der Stakeholderperspektive neue organisationszentrierte Ansätze hervorgebracht (vgl. z.B. Grunig \& Hunt, 1984; Karmasin, 2015), die der zweiseitigen, „symmetrischen“ (Grunig \& Hunt, 1984) Kommunikation zwischen Unternehmen und Anspruchsgruppen Rechnung tragen, um die unterschiedlichen Ansprüche und Interessenlagen „kommunikativ miteinander aus[zu]balancieren und [zu] harmonisieren“ (Wehmeier, 2015: 157). Systemtheoretische Ansätze der PR-Forschung (Szyszka, 2009; Röttger, Preusse \& Schmitt, 2014), sehen im „Beobachten“ und im Reflektieren der eigenen Organisation, aber auch der „Fremdbeobachtungen und -beschreibungen“ die Aufgabe der PR. Übergeordnetes Ziel ist dabei, die „Legitimität“ des Unternehmens zu sichern (Röttger, Preusse \& Schmitt 2014: 127).

Einen Schritt weiter geht der diskurstheoretische Ansatz von Motion \& Leitch (2007), die PR-Schaffende weder als Kommunikationsmanager noch als neutrale 
Mittler betrachten, sondern als „discourse technologists“, die über ihre Kommunikationsarbeit Macht-Wissen-Prozesse im Unternehmen und in der Öffentlichkeit verändern (Motion \& Leitch, 2007: 265). Die “organizational studies” (vgl. dazu Schoenenborn \& Wehmeier, 2014; Cooren et al., 2011) schliesslich fokussieren generell weniger auf die Rolle von Kommunikation als vielmehr auf Prozesse und Praktiken des Kommunizierens, die Unternehmen erst konstituieren.

\section{Transdisziplinäre Perspektive auf das Kommunikationsmanagement}

Diese theoretischen Beiträge zum Fach „Organisationskommunikation“ (Weder, 2010: $11 \mathrm{ff}$.) adressieren aus je unterschiedlichen disziplinären Perspektiven spezifische Funktionen und Herausforderungen des Kommunikationsmanagements. In der Praxis lassen sich diese jedoch nur schwer voneinander trennen; hier befindet sich das Kommunikationsmanagement im ständigen Spannungsfeld zwischen Unternehmenspolitik, Organisation und Öffentlichkeit und ist mit Entscheidungs-, Umsetzungs- und Durchsetzungsproblemen beschäftigt. Zwischen wissenschaftlicher Abstraktion und praktischer Umsetzung haben Theorien und Modelle mittlerer Reichweite (Merton, 1995) anzusetzen, die gemeinsam mit der Praxis sowie über und für diese im Sinne eines transdisziplinären Prozesses (Cameron et al., 1992) entwickelt werden müssen.

Im Kontext des Executive-Master-Studiengangs Communication Management and Leadership (MAS CML), der seit 2004 vom IAM der ZHAW Zürcher Hochschule für Angewandte Wissenschaften verantwortet wird, stehen die Autorin und der Autor dieses Beitrags über ihre Lehrtätigkeit im ständigen Austausch mit den Masterstudierenden, die alle über langjährige Berufspraxis und erste Führungsverantwortung in der Kommunikation verfügen. In allen Jahrgängen haben die Weiterbildungsstudierenden in Diskussionen immer wieder artikuliert, dass ihre Stellung innerhalb der Organisation schwierig sei. Dabei kristallisierten sich vier Problembereiche heraus: Zum einen sind Kommunikationsverantwortliche organisationsintern mit Zielen konfrontiert, die nicht oder nicht ausschliesslich mit strategischer Kommunikation erreicht werden können. Zum andern sind sie zu wenig institutionalisiert in strategische Unternehmensprozesse eingebunden. Hinzu kommt ein Verständigungsproblem im Austausch zwischen Management und Kommunikationsabteilung, da man nicht „die gleiche Sprache spreche“. Und schliesslich sehen sich Kommunikationsverantwortliche Erwartungen von Medien und Bezugsgruppen bezüglich Kommunikationsinhalten und -kanälen gegenüber, die sie oft nicht erfüllen können. Diese komplexe Problemlage wurde 
im Rahmen eines „transdisziplinären Forschungsprogramms“ (Heintel, 2009: 191) bearbeitet. Ziel war es, ein Framework für das Kommunikationsmanagement $\mathrm{zu}$ entwickeln, das eine „Brücke zwischen wissenschaftlicher Abstraktion und praktischer Umsetzung“ (Röttger, 2017: V) schlägt und sowohl in der Aus- und Weiterbildung von KommunikatorInnen als auch in der forschungsbasierten kommunikationsstrategischen Beratung von Unternehmen eingesetzt werden kann.

\section{Modell des identitätsorientierten Kommunikationsmanagements als Ergebnis transdisziplinärer Forschung}

Angelehnt an die PR-theoretischen Ansätze (Röttger, Preusse \& Schmitt, 2014; Szyszka, 2009) hat die institutionalisierte Kommunikation in dem von der Autorin und dem Autor entwickelten Modell des identitätsorientierten Kommunikationsmanagements ${ }^{2}$ das übergeordnete Ziel, für das Unternehmen Legitimität $\mathrm{zu}$ erzeugen und diese zu stabilisieren. Legitimität ist eine Grundvoraussetzung für Reputation, wobei Reputation als öffentlich verhandelte Bewertung in Bezug auf Kompetenz, Integrität und Attraktivität (Eisenegger \& Imhof, 2009) verstanden wird. Reputation entsteht in der Kommunikationsarena, in der sich auch die Images innerhalb einer Bezugsgruppe - etwa Kunden oder Mitarbeitende ausprägen. Ihren positiven Einfluss auf die Reputation entfalten diese Images, wenn sie grundsätzlich konsistent und über alle Bezugsgruppen hinweg gesehen zumindest in den legitimitätssichernden Aspekten kongruent sind (Niederhäuser \& Rosenberger, 2017: $118 \mathrm{ff}$.).

Es lohnt also, das Zustandekommen und die Wirkmechanismen konsensual erzeugter Images einer genaueren systematischen Analyse zu unterziehen. Einerseits werden Images und Reputation geprägt durch direkte Kontakte mit dem Leistungsangebot, den Mitarbeitenden, der Symbolik sowie der Kommunikation des Unternehmens. Andererseits wirken sich auch Kommunikationsbeiträge anderer Akteure über das Unternehmen auf die Fremdwahrnehmung aus. Die direkten Erfahrungen der Bezugsgruppen mit dem Unternehmen sind im Modell (vgl. Abb. 1) als Ergebnisse der Austauschprozesse zwischen Unternehmen und Kommunikationsarena dargestellt. In diesen Interaktionen, die sich meist sprachlich realisieren, wird ein Unternehmen erst sicht- und als von der Umwelt abgrenzbar erfahren. In dem hier vorgestellten Framework wird das, worüber ein

2 Ein ausführlicher Beschrieb des Modells findet sich in Niederhäuser \& Rosenberger, 2017: 7-16. 
Unternehmen wahrnehmbar wird, als Identität bezeichnet. Diese manifestiert sich in den vier Dimensionen Leistungsangebot, Verhalten einzelner Mitarbeitender, multisensorische Symbole wie Architektur, Corporate Design oder Corporate Sound sowie institutionalisierte Kommunikation (Marketingkommunikation, Finanzkommunikation, PR etc.). Über die nicht institutionalisierte, organisationale Kommunikation der Mitarbeitenden und über die Führungskommunikation wird die Identitätsdimension Verhalten manifest.

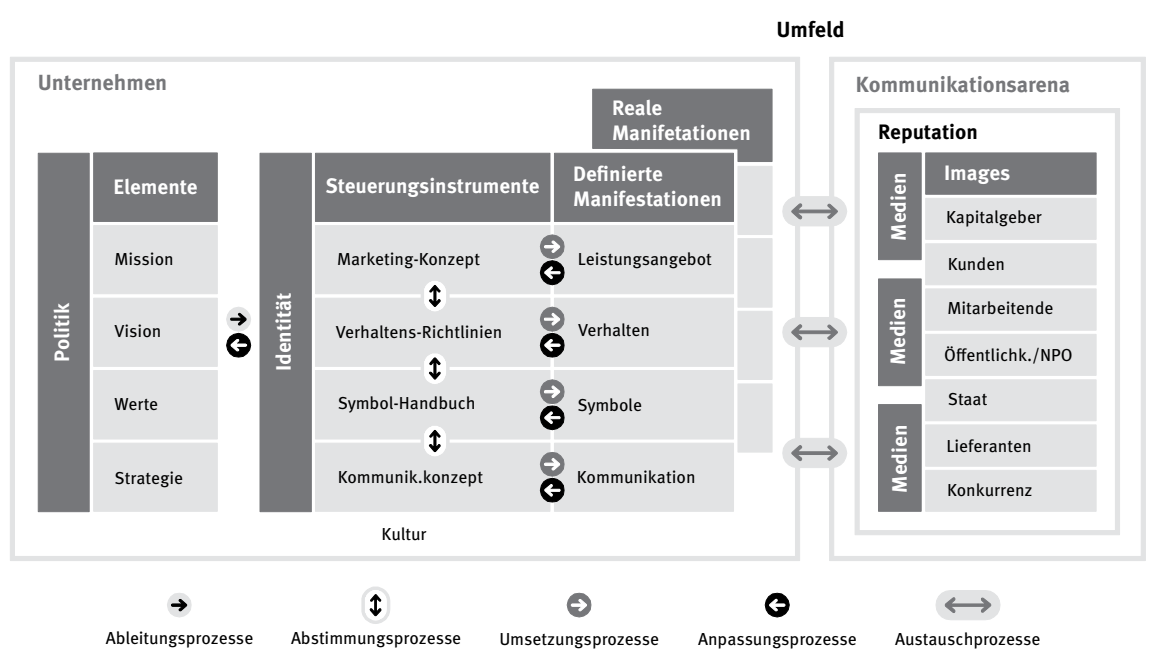

Abb. 1: Modell des identitätsorientierten Kommunikationsmanagements (Niederhäuser \& Rosenberger, 2017: 14)

Das Modell visualisiert zunächst die definierte Identität. Sie umfasst einerseits Steuerungsinstrumente wie Marketingkonzept, Verhaltensrichtlinien, Symbolhandbuch und Kommunikationskonzept, aber auch deren idealtypische Umsetzungen, die definierten Manifestationen. Aus Sicht der Unternehmensführung stellen die Steuerungsinstrumente eine Ableitung aus den auf Stufe Unternehmenspolitik definierten Elementen Mission, Vision, Werte und Strategie dar. In dieser Perspektive ist Identität als handlungsorientierte Umsetzung der Unternehmenspolitik in den vier Dimensionen zu verstehen. Dabei differenziert das Modell zwischen den über die Steuerungsinstrumente definierten Identitätsmanifestationen und den effektiv realisierten und $\mathrm{zu}$ beobachtenden, den sogenannten realen Identitätsmanifestationen. Negativ auf die Reputation wirkt sich zum einen eine zu grosse Diskrepanz zwischen definierter und meist auch kommunizierter Identität und realen Identitätsmanifestationen aus. Ebenfalls negativ zu Buche schlägt die Inkompatibilität der kommunizierten und/oder 
realen Manifestationen mit den Werten und Interessen von Bezugsgruppen oder wenn Widersprüche zwischen den Identitätsmanifestationen sichtbar werden.

Dem Kommunikationsmanagement kommen in Bezug auf das Identitätsmanagement drei Rollen zu: Erstens konstituiert es Identität - wie die anderen drei Identitätsdimensionen. Zweitens hat die autorisierte Kommunikation die Aufgabe, sowohl die Unternehmenspolitik als auch die definierte Identität nach innen und aussen zu vermitteln. Und drittens übernimmt sie zentrale Aufgaben in der Moderation des internen Identitätsdiskurses, wenn es um die Abstimmung zwischen den vier Dimensionen geht.

Das Modell setzt das Kommunikationsmanagement damit bewusst in Relation zu den Bezugsgrössen Unternehmensführung, Organisation und Öffentlichkeit. Darüber hinaus bildet es die unterschiedlichen kommunikativen Prozesse ab, die sich aus diesem komplexen Bezugssystem ergeben: Es geht um Vertextung und Visualisierung von Kommunikationsstrategien in den Ableitungsprozessen, um Realisierung von Identität über Sprache in den Umsetzungsprozessen, um das sprachliche Organisieren von Austauschprozessen und das Erfassen und Bewerten von Texten über das Unternehmen innerhalb dieser Austauschprozesse. Das Kommunikationsmanagement ist aber auch zuständig für die Übersetzung des Beobachteten in- und ausserhalb des Unternehmens in die Sprache der Management-Organisation in den rückkoppelnden Anpassungsprozessen auf Stufe Kommunikation oder auf der Ebene der Unternehmenspolitik. Und schliesslich obliegt ihm die diskursive Verständigung innerhalb der Organisation im Rahmen der Abstimmungsprozesse.

\subsection{Prozess und Methoden der Modellentwicklung}

Aus den von den Weiterbildungsstudierenden thematisierten Problemstellungen entwickelten die Autorin und der Autor in einem ersten Schritt einen Modellentwurf, derverschiedene disziplinäre Ansätze und Erkenntnisse empirischer Studien berücksichtigte. Diese erste Version wurde anschliessend im Rahmen eines Workshops mit Kommunikationsverantwortlichen verschiedener Unternehmen diskutiert und geschärft. In einer nächsten Phase wurden Einzelfallanalysen von Unternehmen gemacht: Mittels halbstandardisierten Leitfadeninterviews mit Geschäftsleitungsmitgliedern und Kommunikationsverantwortlichen der untersuchten Unternehmen sowie mit qualitativen Dokumentenanalysen wurde untersucht, welche Rolle das Kommunikationsmanagement in den jeweiligen Unternehmen spielt, wie es in die Prozesse der Entwicklung und Umsetzung von unternehmenspolitischen Entscheidungen involviert ist und wie die Kommunikation Austausch- und Beobachtungsprozesse führt. 
Die gewonnenen Erkenntnisse wurden in Fallbeschrieben festgehalten (Beispiele in Niederhäuser \& Rosenberger 2011; 2017), wobei das Hauptaugenmerk jeweils auf der Darstellung der unternehmensspezifischen Ausprägungen des identitätsorientierten Kommunikationsmanagements lag. Die Fallbeschriebe stellen damit keine idealtypischen Fälle dar. Vielmehr ermöglichen sie es im Sinne transdisziplinären Arbeitens, Ähnlichkeiten und Differenzen zu erkennen und so ein verallgemeinertes Modell zu erarbeiten, das wiederum für den Einzelfall genutzt werden kann (Krohn, 2008: 66). Ein wesentliches Kennzeichen transdisziplinären Forschens ist der punktuelle Einbezug von Personen aus dem Kreis der Anwender (Defila \& Di Giulio, 2015: 71). Dabei gilt es, Konsens bezüglich der gemeinsamen Fragestellung und der Ziele zu erreichen, die Ergebnisse gemeinsam zu erarbeiten und so aufzubereiten, dass sie von den Anwendern genutzt werden können (Defila \& Di Giulio, 2015: 71f.).

Transdisziplinäre Forschung braucht dialogische Verfahren wie Workshops, Gruppendiskussionen oder Experteninterviews, nicht zuletzt auch um eine gemeinsame Sicht und Sprache zu entwickeln. Sie ist deshalb stets mit terminologischen, text- und gesprächslinguistischen sowie kommunikationspragmatischen Fragestellungen konfrontiert. Methoden und Erkenntnisse der Angewandten Linguistik sollten in den verschiedenen Verständigungsprozessen denn auch gezielt dazu eingesetzt werden, Konsens herzustellen, Anwender zu integrieren und die Diffusion des Erarbeiteten zu befördern.

\section{Literaturverzeichnis}

Bruhn, M. (2014). Unternehmens- und Marketingkommunikation. Handbuch für ein integriertes Kommunikationsmanagement. 3., vollständig überarbeitete Aufl. München: Vahlen.

Cameron, D., Fazer, E., Rampton, B. \& Richardson, K. (1992). Researching language. Issues of power and method. London: Routledge.

Cooren, F., Kuhn, T.R., Cornelissen, J.P. \& Clark, T. (2011). Communication, Organizing and Organization: an Overview and Introduction to the Special Issue. Organization Studies, 32(9), 1149-1170.

Delfia, R. \& Di Giulio, A. (2015). Methodische Gestaltung transdisziplinärer Workshops. In Niederberger, M. \& Wassermann, S. (Hrsg.). Methoden der Experten- und Stakeholdereinbindung in der sozialwissenschaftlichen Forschung (S. 69-93). Wiesbaden: VS Springer.

Einwiller, S. (2014). Reputation und Image: Grundlagen, Einflussmöglichkeiten, Management. In Zerfass, A. \& Piwinger, M. (Hrsg.). Handbuch Unternehmenskommunikation: Strategie Management - Wertschöpfung. 2., vollständig überarbeitete Aufl. (S. 371-391). Wiesbaden: Springer.

Eisenegger, M. \& Imhof, K. (2009). Funktionale, soziale und expressive Reputation - Grundzüge einer Reputationstheorie. In Röttger, U. (Hrsg). Theorien der Public Relations. Grundlagen und Theorien der PR-Forschung. 2., aktualisierte und erweiterte Aufl. (S. 243-264). Wiesbaden: VS Verlag für Sozialwissenschaften. 
Eisenegger, M. (2005). Reputation in der Mediengesellschaft: Konstitution - Issues Monitoring Issues Management. Wiesbaden: VS Verlag für Sozialwissenschaften.

Grunig, J.E., Hunt, T. (1984). Managing public relations. New York: Wadsworth Publishing. Heintel, P. (2009). Wege aus der Randständigkeit - ein Brückenschlag. In Hanschitz, R.-Ch., Schmidt, E. \& Schwarz, G. (Hrsg.). Transdisziplinarität in Forschung und Praxis. Chancen und Risiken partizipativer Prozesse (S. 23-197). Schriften zur Gruppen- und Organisationsdynamik 5. Wiesbaden: VS-Verlag.

Karmasin, M. (2015). PR im Stakeholder-Ansatz. In Fröhlich, R., Szyszka, P. \& Bentele, G. (Hrsg.). Handbuch der Public Relations. Wissenschaftliche Grundlagen mit Lexikon. 3., überarbeitete und erweiterte Aufl. (S. 341-355). Wiesbaden: Springer VS.

Krohn, W. (2008). Epistemische Qualitäten transdisziplinärer Forschung. In Bermann, M. \& Schramm, E. (Hrsg.). Transdisziplinäre Forschung. Integrative Forschungsprozesse verstehen und bewerten. (S. 39-67). Frankfurt a.M.: Campus Verlag.

Merton, R.K. (1995). Soziologische Theorie und soziale Struktur. Berlin: de Gruyter.

Motion, J. \& Leitch, S.R. (2007). A toolbox for public relations: the oeuvre of Michel Foucault. Public Relations Review 33(3). (S.263-268).

Niederhäuser, M. \& Rosenberger, N. (2017). Unternehmenspolitik, Identität und Kommunikation. Modell - Prozesse - Fallbeispiele. 2., vollständig überarbeitete und erweiterte Aufl. Wiesbaden: Gabler.

Niederhäuser, M. \& Rosenberger, N. (2011). Unternehmenspolitik, Identität und Kommunikation. Modell - Prozesse - Fallbeispiele. Wiesbaden: Gabler.

Röttger, U. (2017). Geleitwort aus der Wissenschaft. In Niederhäuser, M. \& Rosenberger, N. (Hrsg.). Unternehmenspolitik, Identität und Kommunikation. Modell - Prozesse Fallbeispiele. 2., vollständig überarbeitete und erweiterte Aufl. Wiesbaden: Springer Gabler.

Röttger, U., \& Preusse, J. \& Schmitt, J. (2014). Grundlagen der Public Relations. Eine kommunikationswissenschaftliche Einführung. 2., aktualisierte Auflage, Wiesbaden: VS Verlag.

Schoeneborn, D., Wehmeier, St. (2014). Kommunikative Konstitution von Organisationen. In Zerfass, A. \& Piwinger, M. (Hrsg.). Handbuch Unternehmenskommunikation: Strategie Management - Wertschöpfung. 2., vollständig überarbeitete Aufl. (S. 411-429). Wiesbaden: Springer.

Szyszka, P. (2009). Organisation und Kommunikation: Integrativer Ansatz einer Theorie zu Public Relations und Public Relations Management. In Röttger, U. (Hrsg.). Theorien der Public Relations. Grundlagen und Perspektiven der PR-Forschung. 2., aktualisierte und erweiterte Aufl. (S. 135-150). Wiesbaden: VS Verlag für Sozialwissenschaften.

Weder, F. (2010). Organisationskommunikation und PR. Stuttgart: UTB.

Wehmeier, St. (2015). Organisationsbezogene Ansätze. In Fröhlich, R., Szyszka, P. \& Bentele, G. (Hrsg.). Handbuch der Public Relations. Wissenschaftliche Grundlagen mit Lexikon. 3., überarbeitete und erweiterte Aufl. (S. 155-170). Wiesbaden: Springer VS.

Zerfass, A. (2010). Unternehmensführung und Öffentlichkeitsarbeit. Grundlegung einer Theorie der Unternehmenskommunikation und Public Relations. 3., aktualisierte Aufl. Wiesbaden: VS Verlag für Sozialwissenschaften.

Zerfass A., Moreno, A., Tench, R., Verčič, D. \& Verhoeven, P. (2008 ff.). European Communication Monitor. Brussels: EACD/EUPRERA. Quadriga Media Berlin. 